\title{
Diferencias en memoria y funciones ejecutivas en niños con diferente nivel lector de Huancavelica y Lima- Callao
}

\author{
Differences in memory and executive functions in children with different \\ level of reading of Huancavelica and Lima - Callao
}

\author{
Ricardo Canales Gabriel ${ }^{1}$, Esther Velarde Consoli ${ }^{2}$ \\ Universidad Nacional Mayor de San Marcos
}
Katherine Susana Lingán Huamán ${ }^{3}$, Juan Ramírez Mendoza
Universidad San Ignacio de Loyola

Recibido: $02-12-19$

Aceptado: $17-12-19$

\begin{abstract}
Resumen
El estudio buscó determinar si existen diferencias en memoria de trabajo y funciones neuropsicológicas ejecutivas en niños con diferente nivel lector pertenecientes a realidades socioculturales distintas. La muestra fue de 71 alumnos de Huancavelica y 82 alumnos de Lima y Callao del 3er. Y 4to. grado de primaria, todos de Instituciones Educativas Públicas.
\end{abstract}

Fue un estudio descriptivo comparativo. Primero se evaluaron los procesos de la lectura en los grupos de Huancavelica y Lima- Callao con el PROLEC- R (Cuetos, Rodríguez, Ruano y Arribas, 2007), encontrándose diferencias significativas. Sobre esa base se procedió a evaluar la Memoria de trabajo, con subtests de la Prueba WISC- IV (Test de Inteligencia de Wechsler-2006) y las funciones neuropsicológicas ejecutivas con algunos tests del BANFE (Flores-Ostrosky, 2012) en ambos grupos.

Los resultados mostraron diferencias significativas en Memoria de Trabajo a favor del grupo de alumnos de Lima- Callao. No se hallaron diferencias significativas en funciones neuropsicológicas ejecutivas. Finalmente, no se halló correlación entre los procesos de la lectura y funciones neuropsicológicas ejecutivas. Se discuten los resultados y sus implicaciones.

Palabras clave: Néstor M.funciones neuropsicológicas ejecutivas; memoria de trabajo; procesos de la lectura.

\footnotetext{
1 Docente principal e investigador de la Facultad de Psicología de la Universidad Nacional Mayor de San Marcos. E-mail: rcanalesg@unmsm.edu.pe

2 Docente principal e investigadora de la Facultad de Educación en la Universidad Nacional Mayor de San Marcos. E-mail: emvelarde@hotmail.com

3 Docente de la Universidad "San Ignacio de Loyola". E-mail: ksusanalingan39@gmail.com

(C) Los autores. Este artículo es publicado por la Revista de Investigación en Psicología de la Facultad de Psicología, Universidad Nacional Mayor de San Marcos. Este es un artículo de acceso abierto, distribuido bajo los términos de la licencia Creative Commons Atribucion - No Comercia_Compartir Igual 4.0 Internacional. (http://creativecommons.org/licenses/by-nc-sa/4.0/) que permite el uso no comercial, distribución y reproducción en cualquier medio, siempre que la obra original sea debidamente citada.
} 


\begin{abstract}
The study sought to determine if there are differences in working memory and executive neuropsychological functions in children with different reading levels belonging to different socio-cultural realities. The sample was 71 students from Huancavelica and 82 students from Lima and Callao from the 3rd. And 4 to. Primary grade, all of Public Educational Institutions.

It was a comparative descriptive study. First, the reading processes in the Huancavelica and Lima-Callao groups were evaluated with the PROLEC-R (Cuetos, Rodríguez, Ruano and Arribas, 2007), finding significant differences. On that basis, the Working Memory was evaluated, with subtests of the WISC-IV Test (Wechsler Intelligence Test-2006) and the executive neuropsychological functions with some BANFE tests (Flores-Ostrosky, 2012) in both groups.

The results showed significant differences in Working Memory in favor of the Lima-Callao student group. No significant differences were found in executive neuropsychological functions.

Finally, no correlation was found between the processes of reading and executive neuropsychological functions.
\end{abstract}

The results and their implications are discussed.

Keywords: Executive neuropsychological functions; working memory; reading processes.

Un importante tema de debate científico en el campo de estudio del aprendizaje de la lectura, es el relativo a cuales serían los procesos cognitivos y psicolingüísticos que subyacen a las deficiencias lectoras. La psicología cognitiva y la psicopedagogía, ha avanzado en los últimos tiempos en el estudio de los problemas de aprendizaje: fracasos en el aprendizaje de la lectura, escritura, matemáticas (Bravo, 2008). Cuetos, Rodríguez, Ruano y Arribas (2007) investigaron respecto a los procesos psicológicos que intervienen en el aprendizaje de la lectura y publicaron una batería para examinar en detalle los procesos perceptivos, léxicos, sintácticos y semánticos, que intervienen en dicho aprendizaje y que al verse afectados no les permiten a los niños adquirir y aprender en los tiempos esperados dichas competencias básicas (Velarde, 2001, Velarde y Canales, 2013).

De otro lado, en los últimos años también se ha prestado atención a los factores socio- económicos y socio- culturales que puedan influir en dicho desarrollo. Son muchos, los niños y adolescentes del interior del país, que crecen bajo condiciones disortogénicas: extrema pobreza, desnutrición marcada, marginalidad, analfabetismo real, bilingüismo (González, 2006). Todo ello va afectando su desarrollo cognitivo, psicolinguístico y social. Dioses (2010) en su estudio con alumnos de tercero de primaria Piura y Lima encontró rendimientos con diferencia significativa a nivel léxico, en la organización sintáctica y en lo 
semántico, en los estudiantes de acuerdo en función a su estrato social y económico. Velarde y Canales (op cit) en el estudio sobre lectura en niños del $1^{\circ}$ al $6^{\circ}$ grado de primaria del Callao, hallaron diferencias significativas según el nivel socioeconómico en los procesos básicos que intervienen en la lectura: perceptivos, como identificación de letras y fonemas, en los procesos léxicos y en los procesos sintácticos y semánticos.

Pero además de los procesos cognitivos y psicolingüísticos mencionados y la condición socio- cultural, también se viene tomando en cuenta a la memoria, particularmente, la memoria de trabajo y los factores neuropsicológicos.

En el estudio de la memoria, al modelo inicial de Atkinson y Shiffrin que en 1968 postulaba que existían aparte del registro sensorial, dos almacenes, uno, el de memoria de corto plazo, y otro el de largo plazo, se agregaron en los años siguientes los modelos basados principalmente en los trabajos de Baddeley \& Hitch (1974) tratando de dar cuenta cómo es que la información podía pasar de un almacén al otro, bajo el concepto de memoria de trabajo. Dicho modelo fue reformulado dos décadas después (Baddeley, 2000) cuando se planteó que la estructura y funcionamiento de la memoria de trabajo se basaba en cuatro componentes: bucle fonológico, agenda viso-espacial, el sistema ejecutivo central y el buffer episódico. El bucle fonológico permite mantener la información auditiva por unos segundos en la mente hasta que esta pueda ser procesada por el cerebro. La agenda visoespacial tiene un funcionamiento similar pero se basa en información visual. El bufer episódico integra toda la información desde la memoria de corto plazo y permite el paso a memoria de largo plazo, y el ejecutivo central organiza y supervisa el funcionamiento de los cuatro componentes.

Basándose en este modelo, Brito (2017) sostiene la importancia que la memoria de trabajo tiene no solo para la conducta en general, sino también para las actividades de aprendizaje, entre ellas, principalmente la lectura.

Manga y Ramos (1991) usando la Batería neuropsicológica Luria- DNI, examinaron funciones neuropsicológicas en los niños: funciones motoras y sensoriales, funciones del lenguaje oral, lectura, escritura, cálculo y memoria. Encontró que los alumnos con dislexia rendían muy por debajo de los que tenían un nivel de lectura dentro del promedio, en particular en rendimiento lector, lenguaje escrito, procesamiento fonológico y memoria.

Canales (2012) utilizando la Prueba de evaluación neuropsicológica infantil (Quintanar y Solovieva 2004) y la prueba Procesos de la lectura-R (Cuetos 2007), encontró que los niños que obtenían las puntuaciones más bajas en los factores neuropsicológicos de planeamiento, estructuración del movimiento, funciones práxicas y gnosias, procesos percepto- visuales, memoria audioverbal y capacidad 
audio- fonémica, eran los que en su mayoría presentaban problemas con el aprendizaje de la lectura.

Ampliando el enfoque, y tomando en cuenta la posible relación entre condiciones externas, sociales y culturales, con el desarrollo de las funciones neuropsicológicas habría que considerar los aportes de Changeux (1983) en su planteamiento sobre el desarrollo epigenético, remarcando la importancia del medio ambiente para el desarrollo neuronal y la organización cerebral. Igualmente, Ortiz (2004) ha planteado la importancia de considerar en el desarrollo psíquico y la estructuración y maduración neocortical, además de los aspectos epigenéticos, también los sociocinéticos.

Respecto a las funciones ejecutivas, se estudia la relación entre nivel socioeconómico y desarrollo dichas funciones (Arán, 2011), el desarrollo de funciones ejecutivas en contextos bilingües (Martínez y Henao, 2006) y las funciones ejecutivas en estudiantes de universidades públicas de 3 regiones del país: Lima, Huancavelica y Ayacucho y (Canales, Velarde, Lingán y Echavarría, 2017). En el caso de estos dos últimos grupos, los alumnos expuestos muy temprano a una situación socio- cultural interlectal y al bilingüismo quechua- castellano mostraban menores niveles de funcionamiento más bajo en las funciones neuropsicológicas ejecutivas.

En función a estas orientaciones la investigación se planteó como objetivo establecer si existirían diferencias en el rendimiento a nivel de memoria de trabajo y las funciones neuropsicológicas ejecutivas, en niños con adecuado nivel lector comparados a niños con deficiencias lectoras, que crecen en ambientes socioculturales distintos.

\section{METODO}

Se trata de un estudio descriptivo comparativo, y además correlacional, en donde en forma transversal, se recolectan datos sobre memoria de trabajo y funciones neuropsicológicas ejecutivas buscando establecer las diferencias, y de otro lado estudiar las posibles correlaciones entre procesos de lectura y funciones neuropsicológicas ejecutivas, considerando su pertenencia a realidades socioculturales distintas.

\section{Participantes}

La población estuvo constituida por los niños de tercero y cuarto grado de primaria de Instituciones Educativas públicas perteneciente al nivel socioeconómico bajo y de zona rural de la comunidad de Pasos, provincia de Tayacaja en Huancavelica: 74 niños, y alumnos de Instituciones Educativas públicas perteneciente al nivel socioeconómico bajo de Lima (San Juan de Miraflores) y Callao (Pachacútec): 237. 
Los participantes fueron seleccionados teniendo en cuenta los estudios previos (Canales, et al, 2012, 2017a, 2017b, 2017c) y manteniendo el criterio de representatividad. La muestra se escogió de modo intencional, considerando la pertenencia a Instituciones educativas públicas del ámbito geográfico establecido, la edad y el grado escolar, así como su pertenencia al mismo nivel socio- económico. El criterio diferenciador fue en torno a las distintas realidades socioculturales, (Sánchez y Reyes 2002).

Finalmente, la muestra quedó constituida así:

Huancavelica: 71 alumnos de $3^{\circ}$ y $4^{\circ}$ grado de primaria, pertenecientes a la Institución educativa 30961, "José Antonio Encinas" de la Comunidad de Pasos y a la Institución Educativa $N^{\circ} 31208$ de Santa Cruz de Ila, ambos colegios de la Provincia de Tayacaja, Huancavelica.

Lima y Callao: 82 estudiantes de $3^{\circ}$ y $4^{\circ}$ grado de primaria pertenecientes al colegio "Ollantay" de San Juan de Miraflores, Lima, y a la I.E. "Virgen de Guadalupe" de Pachacútec, provincia constitucional del Callao.

La edad promedio fue 9 años en Huancavelica y 9.38 en Lima- Callao.

Se consideraron alumnos de ambos sexos: femenino, $40.8 \%$ en el caso de Tayacaja y $54.9 \%$ en el caso de Lima- Callao; y masculino, $59.2 \%$ en el caso de Huancavelica y $45.1 \%$ en el caso de Lima-Callao.

Los niños de Huancavelica en un79\% eran castellano hablantes y en un $19.7 \%$ quechua hablantes, mientras que en Lima, el 100\% eran castellano hablantes.

\section{Instrumentos}

1. Memoria de trabajo. Subtests: Letras y números y Memoria de dígitos de la Prueba de Inteligencia de Weschler (2006): WISC-IV: coeficientes de fiabilidad van de o,86 (Velocidad de procesamiento) a 0,95 (CI Total).

Los coeficientes de fiabilidad del subtest Memoria de dígitos es de 0.84; y el de Serie de Letras y números es de 0.84

La Validez de la prueba, se sustenta en las evidencias de contenido de la prueba, procesos de respuesta, estructura interna y relación con otras variables. Respecto a la relación con otras variables, se realizaron diversos estudios para ver la relación entre el WISC-IV y el WISC-III y se hallaron coeficientes de correlación de 0.72 (MT y RP) y 0.89 (CI total y CI total).

BANFE: Batería de funciones ejecutivas de Flores, J. y Ostrosky, F. (2012). Pruebas neuropsicológicas: Stroop, Laberintos, Señalamiento autodirigido, Torres de Hanoi y Memoria viso- espacial. 
Respecto a la confiabilidad, Flores (2014), sostiene refiere una concordancia entre aplicadores de 0,80 .

Sobre la Validez, esta batería, posee:

- Validez de constructo, Validez clínica y corroborado por estudios de neuroimagen funcional. en tanto son pruebas que han surgido del campo de la neuropsicología para el estudio del funcionamiento del lóbulo frontal y han demostrado que son sensibles y especificas para dicha labor.

3. Prueba para evaluar la lectura: PROLEC-R (Cuetos et al, 2007) que evalúa aspectos perceptivos, reconocimiento de palabras, procesos sintácticos y semánticos que intervienen en la lectura. La fiabilidad de la prueba, se estableció en base al estudio de su consistencia interna, con el coeficiente alfa de Cronbach. Los valores alcanzados fueron satisfactorios: 0.79 Respecto a la Validez del PROLEC R, y en relación a su validez de criterio se tomó en cuenta la opinión de profesores respecto al rendimiento de los alumnos en lectura. Las correlaciones halladas fueron significativas al nivel $\mathrm{p}<0,001$

\section{Procedimiento}

El procedimiento general para realizar la investigación fue:

- Instrucción y entrenamiento a los docentes y alumnos que forman el grupo de investigación en la evaluación de los aspectos cognitivos relacionados con la Memoria de trabajo y la velocidad de procesamiento de la información; así como en las pruebas neuropsicológicas.

- Selección de la muestra con los criterios señalados, realizándose las coordinaciones institucionales del caso.

- La evaluación se realizó de forma individual, lo cual significó un mayor tiempo invertido en el estudio. Los evaluadores a la hora de plantear los ejercicios solo continuaron adelante, cuando confirmaron que el alumno había comprendido la tarea.

- Terminadas de aplicar las pruebas, se recopiló toda la información, se preparó la data y se hicieron los análisis que corresponden.

Concordante con uno de los objetivos de la investigación, se utilizaron técnicas comparativas de medias para grupos independientes (prueba "t"), y también tomando en cuenta otro de los objetivos, que era el tratar de establecer el grado de asociación entre los factores neuropsicológicos ejecutivos y los procesos 
de la lectura, se utilizó para ver el índice de correlación el coeficiente $r$ de Pearson. Esto se hizo a un nivel de significación del 0,05

\section{RESULTADOS}

Se muestran las diferencias en rendimiento lector considerando su realidad sociocultural:

Tabla 1

Diferencias en los procesos de la lectura.

\begin{tabular}{lcccccccc}
\hline & \multicolumn{3}{c}{ Huancavelica } & \multicolumn{2}{c}{ Lima } & \multirow{2}{*}{$\mathbf{t}$} & $\mathbf{p}$ & \multirow{2}{*}{$\mathbf{d}$} \\
\cline { 2 - 5 } & $\mathbf{M}$ & $\mathbf{D E}$ & $\mathbf{M}$ & $\mathbf{D E}$ & & & \\
\hline Identificación de letras & 15.56 & 3.00 & 18.00 & 1.85 & -5.941 & .000 & 0.97 \\
Identificación de diferencias & 17.25 & 2.53 & 18.56 & 1.56 & -3.776 & .000 & 0.62 \\
Lectura de palabras & 37.62 & 4.28 & 38.93 & 1.67 & -2.419 & .018 & 0.40 \\
Lectura de pseudopalabras & 35.99 & 4.72 & 37.38 & 2.73 & -2.188 & .031 & 0.36 \\
\hline
\end{tabular}

Aparecen diferencias estadísticamente significativas entre los alumnos de distinta realidad socio- cultural, en los procesos evaluados concernientes a la lectura. Al examinar el tamaño del efecto, se encuentra que la magnitud de las diferencias es grande en la tarea de identificación de letras, moderada en la tarea de identificación de diferencias entre pares de palabras, y pequeña en las tareas de lectura de palabras y lectura de pseudopalabras. Los estudiantes de Lima- Callao, logran mayores puntajes que los niños de Huancavelica.

Para los fines del presente estudio, se consideró en conjunto a los niños de Huancavelica como grupo con deficiencias en los procesos básicos de la lectura; y a los niños de Lima- Callao, como grupo con rendimiento regular o promedio, de acuerdo a la edad y el grado escolar.

A continuación, se muestran las diferencias entre los niños lectores normales y los niños con deficiencias lectoras en memoria de trabajo:

Tabla 2

Diferencias según los resultados obtenidos en las tareas de memoria de trabajo.

\begin{tabular}{|c|c|c|c|c|c|c|c|}
\hline & \multicolumn{2}{|c|}{ Huancavelica } & \multicolumn{2}{|c|}{ Lima } & \multirow{2}{*}{$t$} & \multirow{2}{*}{$\mathbf{p}$} & \multirow{2}{*}{ d } \\
\hline & M & DE & M & DE & & & \\
\hline Retener dígitos (forma directa) & 6.22 & 1.59 & 6.88 & 1.49 & -2.657 & .009 & 0.42 \\
\hline Retener dígitos (forma inversa) & 4.97 & 1.34 & 5.54 & 1.29 & -2.666 & .008 & 0.43 \\
\hline $\begin{array}{l}\text { Ordenamiento y retención de } \\
\text { series de dígitos y letras }\end{array}$ & 10.26 & 3.91 & 12.29 & 3.62 & -3.359 & .001 & 0.54 \\
\hline
\end{tabular}


Se encontraron diferencias estadísticamente significativas en las tareas vinculadas a la memoria de trabajo. Las magnitudes de las diferencias presentan variabilidad entre las tareas: en el ordenamiento y retención de series de dígitos y números se encuentra una magnitud moderada. En las otras tareas que se exponen en la tabla 3, se aprecian diferencias de magnitud pequeña. En todas las tareas son los estudiantes de Lima que obtienen mayores puntuaciones.

Se examina si existen diferencias entre los funciones neuropsicológicas entre los niños lectores normales y los niños con deficiencias lectoras considerando su realidad socio- cultural.

Tabla 3

Diferencias según los resultados en las tareas de funciones neuropsicológicas ejecutivas.

\begin{tabular}{|c|c|c|c|c|c|c|c|}
\hline & \multicolumn{2}{|c|}{ Huancavelica } & \multicolumn{2}{|c|}{ Lima } & \multirow{2}{*}{$t$} & \multirow{2}{*}{$\mathbf{p}$} & \multirow{2}{*}{ d } \\
\hline & M & DE & M & DE & & & \\
\hline Stroop "A" & 78.34 & 13.72 & 75.85 & 16.23 & 1.013 & .312 & 0.16 \\
\hline Stroop “B” & 76.70 & 15.15 & 77.32 & 14.79 & -.253 & .801 & 0.04 \\
\hline Señalamiento autodirigido & 17.00 & 5.74 & 16.12 & 5.82 & .936 & .351 & 0.15 \\
\hline $\begin{array}{l}\text { Memoria de trabajo visoespacial } \\
\text { (secuencia máxima) }\end{array}$ & 1.92 & 1.09 & 1.85 & 0.89 & .386 & .700 & 0.07 \\
\hline $\begin{array}{l}\text { Laberintos } \\
\text { (planeación codificado) }\end{array}$ & 3.97 & 1.76 & 3.77 & 2.00 & .670 & .504 & 0.10 \\
\hline $\begin{array}{l}\text { Torre de Hanoi } \\
\text { (movimientos codificado) }\end{array}$ & 3.44 & 1.77 & 3.49 & 2.20 & -.157 & .876 & 0.02 \\
\hline
\end{tabular}

No se encontraron diferencias estadísticamente significativas en ninguna tarea. Al hacer el análisis, se encontró ambos grupos en promedio rinden igual en términos estadísticos. Tanto en lo que corresponde a las tareas que examinan el funcionamiento del área órbito medial frontal (tareas de Stroop A y B, y un aspecto en Laberintos), como en el área dorsolateral de Memoria de trabajo (tareas de Memoria de trabajo viso-espacial y señalamiento autodirigido), como en área dorso lateral de Funciones ejecutivas (tareas de Torres de Hanoi y dos aspectos de Laberintos).

De otro lado, se examinó el grado de asociación entre el desarrollo los procesos de la lectura y las funciones ejecutivas en los estudiantes de escuelas en zona rural con mayor influencia de bilingüismo quechua- castellano.

Dado que en los anteriores análisis se ha demostrado que existen, en las variables vinculadas con el proceso lector, diferencias moderadas y altas en las puntuaciones obtenidas por los estudiantes de Lima y los estudiantes de Huancavelica, se optó por realizar los análisis de correlación para cada subgrupo muestral. 
A partir de los resultados, se examinó si es viable o no determinar cuál de las habilidades vinculadas con las funciones neuropsicológicas ejecutivas explica mejor las puntuaciones obtenidas en los procesos más relevantes de la lectura.

En la tabla 5 apreciamos los resultados del análisis de correlación bivariada de Pearson para explorar las relaciones entre las diferentes variables del estudio en la muestra de los niños de Huancavelica. Como se aprecia, las tareas vinculadas con la lectura no presentan correlaciones estadísticamente significativas con ninguna tarea vinculada a las funciones neuropsicológicas ejecutivas.

Tabla 4

Correlaciones entre las variables de estudio para la muestra de Huancavelica.

\begin{tabular}{lccccccccc}
\hline & 1 & 2 & $\mathbf{3}$ & $\mathbf{4}$ & $\mathbf{5}$ & $\mathbf{6}$ & $\mathbf{7}$ & $\mathbf{8}$ & $\mathbf{9}$ \\
1. ID & 1 & & & & & & & & \\
2. LP & $.327^{* *}$ & 1 & & & & & & & \\
3. LPS & $.399^{* *}$ & $.787^{* *}$ & 1 & & & & & & \\
4. STA & -.136 & .126 & -.019 & 1 & & & & & \\
5. STB & -.049 & .039 & -.129 & $.452^{* *}$ & 1 & & & & \\
6. SAU & .014 & -.003 & -.101 & .123 & .126 & 1 & & & \\
7. MVE & $.298^{*}$ & .042 & .188 & -.182 & $-.367^{* *}$ & -.150 & 1 & & \\
8. LAB & -.078 & -.162 & -.113 & .014 & -.067 & -.145 & .147 & 1 & \\
9. THA & -.178 & .039 & -.066 & .077 & .123 & $.291^{*}$ & .019 & .137 & 1 \\
\hline
\end{tabular}

Nota: Se muestran los coeficientes $r$ de Pearson; $N=73$;

ID = Identificación de diferencias, LP $=$ Lectura de palabras, LPS $=$ Lectura de pseudopalabras, STA $=$ Stroop forma "A", STB = Stroop forma "B", SAU = Señalamiento autodirigido, MVE = Memoria visoespacial, LAB = Laberintos, THA = Torre de Hanoi.

$* \mathrm{p}<.05 ; * * \mathrm{p}<.01$

Tabla 5

Correlaciones entre las variables de estudio para la muestra de Lima.

\begin{tabular}{lccccccccc}
\hline 1. ID & $\mathbf{1}$ & $\mathbf{2}$ & $\mathbf{3}$ & $\mathbf{4}$ & $\mathbf{5}$ & $\mathbf{6}$ & $\mathbf{7}$ & $\mathbf{8}$ & $\mathbf{9}$ \\
2. LP & 1 & & & & & & & & \\
3. LPS & $.276^{*}$ & 1 & & & & & & & \\
4. STA & $.233^{*}$ & $.500^{* *}$ & 1 & & & & & & \\
5. STB & -.028 & -.059 & -.090 & 1 & & & & \\
6. SAU & .051 & .017 & -.065 & .194 & 1 & & & & \\
7. MVE & -.064 & .134 & .059 & -.054 & -.119 & $.322^{* *}$ & 1 & & \\
8. LAB & -.098 & .005 & .091 & .053 & .073 & $-.364^{* *}$ & .087 & 1 & \\
9. THA & .099 & -.071 & .047 & .007 & .164 & $-.298^{* *}$ & -.070 & .203 & 1 \\
\hline
\end{tabular}

Nota: Se muestran los coeficientes $r$ de Pearson; $N=73$; 
ID = Identificación de diferencias, LP = Lectura de palabras, LPS = Lectura de pseudopalabras, STA = Stroop forma "A", STB = Stroop forma "B", SAU = Señalamiento autodirigido, MVE = Memoria visoespacial, LAB = Laberintos, THA = Torre de Hanoi.

Finalmente se examinó si existía correlación entre dichas variables, en la muestra de los niños de Lima.

Como se puede apreciar, no se encontraron correlaciones estadísticamente significativas entre las variables vinculadas a la lectura y las relacionadas con las funciones neuropsicológicas ejecutivas.

\section{DISCUSIÓN}

En primer término, se aprecia que en lo referente a los procesos básicos que intervienen en la lectura: identificación de letras, lectura de palabras y lectura de pseudopalabras, existen diferencias significativas a favor del grupo de LimaCallao. Los niños de Huancavelica, que cuentan con una realidad socio- cultural caracterizada por la condición socio- económica baja, con un marcado grado de marginalidad (zona rural, alejada del circuito económico, comercial y cultural de la capital) y con predominancia de bilingüismo quechua- castellano, presentan deficiencias en el desarrollo de las habilidades básicas para la decodificación lectora.

En términos generales, ello guardaría relación con la disortogénesis en la evolución psicológica del niño peruano (González, 2006; Velarde, et al 2014, Canales 2017): extrema pobreza, marginalidad, bilingüismo quechua- español. En tal sentido, y tal como se señala, los niños de las poblaciones con marginalidad tanto de tipo socio-económica como cultural- lingüística, tendrían un desarrollo psicológico con mayores interferencias que los niños de las poblaciones o comunidades que solo tiene o marginalidad económica o marginalidad lingüística.

De otro lado, se pudo apreciar que en los procesos cognitivos básicos examinados que guardarían relación con la lectura, en este caso Memoria de trabajo, también se muestran diferencias significativas en el rendimiento de los niños de los grupos examinados a favor del grupo de Lima- Callao.

Algunos estudios han referido, que la Memoria de trabajo, está relacionada directamente, no solo con la decodificación, sino también con la velocidad lectora. Canales (2012), en estudio realizado con niños de dos niveles socio- económicos distintos: medio y bajo en el Callao, encontró correlación significativa, entre la memoria verbal y los aspectos semánticos, principalmente en el sector socioeconómico medio. Ello reflejaría la importancia del desarrollo de la capacidad de registrar y almacenar información, tanto por la vía visual y auditiva en el corto plazo, con la memoria semántica (proceso de alto nivel muy importante para la lectura). 
Quizá el punto más interesante en el presente estudio, es que habiéndose hallado diferencias importantes a nivel de procesos en el rendimiento lector, en la memoria de trabajo y en la velocidad lectora, no se hallaron diferencias en el rendimiento a nivel de las funciones ejecutivas entre los niños de Huancavelica y de Lima- Callao.

Ello más aún, considerando que la investigación de Canales et al (2017b) realizado en Ayacucho, si se hallaron diferencias significativas en las funciones neuropsicológicas ejecutivas cuando se examinaron a niños de la comunidad de Socos y de Pilancucho y se les comparó con el rendimiento de Lima y Callao.

Una interpretación al respecto es que a diferencia del referido estudio en que se examinó a alumnos del 5to y 6to grado de primaria con edad promedio 10.83 en Ayacucho, y 10.94 en Lima- Callao; en la presente investigación, se examinó a niños de 3ro y 4to. grado con 9 años en Huancavelica, y 9.38 en Lima- Callao, es decir en términos etáreos había una diferencia de casi dos años, y tal como lo muestran diversos estudios de psicología evolutiva (Piaget, 1981; Vigotski, 1964; Papalia y Wendkos, 1998) dicha diferencia pueden ser muy importante en el desarrollo psíquico y neuropsicológico de los niños.

Flores y Ostrosky (2012) señalaron que ciertas funciones ejecutivas, como la memoria verbal de trabajo, la memoria viso- espacial, la flexibilidad mental, el planeamiento secuencial, se desarrollan a partir de los 12 años, y que también hay funciones neuropsicológicas ejecutivas de aparición tardía, como fluidez verbal, generación de categorías abstractas o la metacognición que se van logrando alrededor de los 16 años; y ello, en buena parte tiene que ver con los procesos neurobiológicos de mielinización de los diversos grupos neuronales en distintas zonas del cerebro.

Rubia, Overmeyer, Taylor, Brammer, Williams y Simmons (2001), sostiene que de modo diferente a lo que ocurre con los animales cuya base neuronal y conexiones interhemisféricas están establecidas cuando nace, en el humano ello lleva mucho tiempo. Las conexiones entre las zonas frontales y las posteriores, así como con zonas subcorticales, continúan durante toda la adolescencia e incluso llegan hasta la tercera década de la vida.

Esta realidad neuropsicológica, iría de la mano con lo que ocurre a nivel cognitivo. Piaget ha demostrado que los niños cuando se aproximan a los 7 años aún se encuentran en el nivel pre- operatorio, es decir su pensamiento está guiado básicamente por la percepción y el enfoque empirista. Necesita un grado de maduración y desarrollo, que está directamente vinculado, al entorno social, cultural y educativo, para que avance hacia el nivel operatorio concreto, es decir, cuando ya se encuentra en condiciones de formar conceptos, ha logrado las conservaciones de la masa o sustancia, del peso, y luego de la velocidad. Lo que 
habría que señalar es que muchos niños en nuestro país, aún teniendo 8 o 9 años, aún no han llegado a dicho nivel de funcionamiento cognitivo lógico operatorio concreto. Precisamente ese es un efecto de la disortogénesis.

En tal sentido, al parecer, los niños examinados, tanto en Huancavelica como en Lima- Callao de 9 años de edad, recién estarían avanzando hacia este nivel cognitivo, y asimismo, las funciones neuropsicológicas ejecutivas aún se encontrarían en ciernes. Ello tiene que ver directamente con la maduración, conformación y funcionamiento de redes neuronales a nivel del lóbulo frontal, que precisamente en el ser humano, es la estructura cerebral de aparición más tardía.

Luria (1979), hablaba de las 3 grandes unidades funcionales del cerebro, la primera referidad al despertar y el estado de alerta dependiente de la activación de la formación reticular; la 2da. Unidad, encargada de recepcionar, procesar y analizar la información que proviene del medio y que depende del funcionamiento de los lóbulos occipitales, temporales y parietales, y la 3era. Unidad funcional, la que se encarga de la planificación, anticipación, verificación y regulación de la conducta, dependientes de los lóbulos frontales. Esta última estructura y sus redes neuronales correspondientes, son las que aparecen más tardíamente en el ser humano, y dependen directamente de lo social, de la cultura y la educación.

Ortiz (op cit) también se refirió con amplitud al punto de la maduración y desarrollo de los lóbulos frontales. Quizá la idea más interesante de dicho autor, sea el señalamiento crítico a los diferentes enfoques sobre el cerebro, que cuando éstos hablan de lo social, simplemente lo hacen como si fuera un entorno, algo externo, pero que no explican cómo lo social está presente al interior del cerebro. Con la tesis de la sociocinesis se explica cómo el sistema nervioso central, el cerebro, y todo el individuo, es necesariamente reorganizado desde lo social y cultural.

Los diversos estudios sobre surgimiento y desarrollo de las funciones ejecutivas: control inhibitorio, memoria de trabajo, flexibilidad mental (Flores y Ostrosky, 2012) indican que el máximo punto de desarrollo se da en torno a los 9 años (especie de "boom" neuropsicológico ejecutivo), es decir por esa época se da un punto de quiebre, un salto cualitativo, que va marcando la diferencia en el desarrollo neuropsicológico de los niños. Luego, la tendencia avanza hacia una estabilización. La cuestión principal en este punto es preguntarnos ¿Por qué ciertos niños, logran dicho punto de quiebre a los 8-9 años, y otros, no lo logran?

La similitud en los resultados alcanzados en funciones ejecutivas de los niños de ambos grupos examinados, pertenecientes al nivel socio- económico bajo (rural y urbano), reflejaría el hecho real de que dichas funciones de alto nivel - muy relacionadas a los procesos cognitivos de abstracción, categorización y metacognición- aún están en ciernes, es decir estarían gestándose desde los 7,8, 
9 años, pero directamente de la mano con el factor socio- cultural. Luego, dichas funciones se iran configurando de mejor modo, a los once o doce años.

A partir de todo ello, estamos en condiciones de afirmar, que dicho salto cualitativo, depende directamente, ya no tanto de la maduración neurobiológica (el "hardware"), sino principalmente de la cultura, la organización y la información social ("software") que rodea al niño. Es en ese sentido que habría que atender con más interés, no tanto al proceso evolutivo, endógeno, del desarrollo de la inteligencia en sí - y de las funciones ejecutivas-; sino al papel que juega el aprendizaje, el rol de la escuela, del maestro, de los adultos, que permiten potenciar, fortalecer y desarrollar las capacidades cognitivas, psicolinguísticas (con las redes neuronales subyacentes) en el niño.

Es en ese punto de inflexión ascendente, en ese necesario tránsito que deben dar los niños de la etapa cognitiva pre operatoria, donde predomina el empirismo y la visión perceptivista de las cosas, hacia una visión conceptual, categorial (el predominio de la razón y las funciones neuropsicológicas de alto nivel), en donde debe entrar a tallar con más fuerza el entorno socio- cultural. Ello implica, el uso y manejo de la lengua, el sistema educativo, la tecnología, el conocimiento científico; y todo ello, internalizado y estructurado en la mente y las redes neuronales del cerebro del niño, lo que explicaría la diferencia entre los grupos humanos, de acuerdo pues a la condición socio- económica, la marginalidad o no, y la presencia de los factores socio-culturales.

Para el logro de habilidades básicas tal como es el aprendizaje de la decodificación lectora, lo básico es la percepción, la memoria de corto plazo, la organización visual- espacial y algunas habilidades psicolinguísticas; pero para avanzar hacia el desarrollo de capacidades como la comprensión lectora, la formación de conceptos, el pensamiento analítico e hipotético deductivo, así como para el desarrollo de capacidades sociales y morales, son indispensables los procesos de alto nivel, y ahí entran a tallar las funciones neuropsicológicas ejecutivas.

El momento crítico que estamos analizando, puede entenderse también como una "ventana de oportunidad", lo que significa que en dicho período $(7,8$, 9 años) tienen que darse las condiciones mínimas para que el cerebro (y sus redes neuronales correspondientes), así como las capacidades cognitivas y funciones neuropsicológicas, se preparen para dar el salto cualitativo en su desarrollo.

Y tal como se ha visto en estudios anteriores, es en dicha etapa, cuando los niños se han ido incorporando al sistema educativo primario, que se van acentuado las diferencias en el desarrollo de las capacidades, entre los niños de las zonas rurales con contextos de bilinguismo y marginalidad, y los niños de las zonas 
urbanas (mas aún si son de condicion socio- económica media o alta) cercanas a los circuitos económicos, culturales y educativos.

A partir de ahí, puede irse visualizando, como, al principio, cuando dichas funciones ejecutivas están empezando a surgir, al comparar niños del mismo estrato socio- económico (rural y urbano), no aparecen las diferencias neuropsicológicas, pero luego, a los 10, 11 o 12 años, se van perfilando las diferencias en dichas funciones. En las zonas rurales, la pobreza extrema, la marginación social, el analfabetismo de los padres, el problema lingüístico quechua- castellano, las deficiencias en términos educativos, etc. irían mostrando su peso en los procesos de maduración y desarrollo de las redes neuronales, en este caso, del lóbulo frontal. Los niños y adolescentes de la capital, ingresando a la adolescencia, verían atenuados dichos efectos.

\section{CONCLUSIONES}

1. Se halló diferencia estadísticamente significativa en identificación de letras, y procesos léxicos, sintácticos y semánticos, en el rendimiento de los niños de Huancavelica comparados con los de Lima- Callao, a favor de los últimos, lo cual podría sugerir que por ser niños pertenecientes a una realidad socio- cultural en donde están presentes diversos factores disortogénicos, pobreza extrema, marginalidad y el problema del bilinguismo quechua- castellano, todo ello podría estar afectando sus proceso del aprendizaje de la lectura en castellano.

2. Se halló diferencia estadísticamente significativa en Memoria de trabajo, en el rendimiento de los niños de Huancavelica comparados con los de Lima- Callao, a favor de los últimos, lo cual es importante tener en cuenta por cuanto dicha capacidad cognitiva se potencia en contacto directo con el aprendizaje y los factores culturales.

3. No se halló diferencia estadísticamente significativa en funciones ejecutivas, en en rendimientos de los estudiantes de Huancavelica comparados con los de Lima- Callao, lo cual indicaría que a nivel neuropsicológico, es decir de organización y desarrollo de las redes neuronales encargadas de las actividades humanas desde las más simples hasta las más complejas, no se aprecian aún los efectos por parte de los factores socio- culturales.

4. No se halló asociación entre procesos que intervienen en la lectura y funciones ejecutivas tanto en los alumnos de Huancavelica como en los de Lima- Callao. 


\section{NOTA DE RECONOCIMIENTO}

Agradecemos a los estudiantes de psicología y educación que colaboraron con el estudio. Estos fueron: de la Facultad de Psicología de la UNMSM, Juan Ramírez, Daniela Castañeda, Flor Bastida, Angélika Pizarro, Andrés Pereyra, Helen Silva y Jorge Luis Torres; y de la Facultad de Educación: Yoly Rodríguez, Anthony Trujillo, Dulce Iglesias, Vanessa Jurado y Anais Pablo.

\section{REFERENCIAS BIBLIOGRÁFICAS}

Arán, V. (2011) Avances en Psicología Latinoamericana/Bogotá (Colombia)/Vol. 29(1)/ pp. $98-113$

Bravo, L. (2008) Psicología de las dificultades del aprendizaje escolar. Santiago de Chile. Edit. Universitaria.

Canales, R. (2012): Asociación entre factores neuropsicológicos, procesos cognitivos y niveles de lectura en niños de diferente nivel socioeconómico del callao. Febreroabril 2011. Tesis de Maestría en Medicina- Neurociencias. Universidad Nacional Mayor de San Marcos. Lima. Perú.

Canales R., Velarde E., Lingán S., Echavarría, L. (2017a) "Funciones ejecutivas y Juicio moral en alumnos pertenecientes a realidades socio- culturales distintas: Lima, Ayacucho. Revista de Investigación en Psicología Vol. 20 - N. 1 - 2017, pp. 43 - 60

Canales R., Velarde E., Lingán S., Ramírez, J. (2017b). Habilidades cognitivas y funciones neuropsicológicas ejecutivas en niños bilingües (lengua nativa- castellano) y monolingües castellano. Lima. Revista de Investigación en Psicología Vol. 20 - N. ${ }^{\circ}$ 2 - 2017, pp. $253-268$

Canales, R. (2017c). Diferencias en lenguaje oral y funciones neuropsicológicas en estudiantes universitarios de Lima, Arequipa, Huancavelica según su realidad social y lingüistica. Perú 2014-2015. Tesis Doctoral en Medicina- Neurociencias. Universidad Nacional Mayor de San Marcos. Lima. Perú.

Cuetos, F., Rodríguez, B., Ruano, E. y Arribas, D. (2007). PROLEC-R: Batería de evaluación de los procesos lectores. Madrid. TEA

Changeux. J.P. (1983). El hombre neuronal. Madrid. Espasa Calpe.

Dioses, A..; Evangelista, C.; Basurto, A. , .T; Morales, Alcántara, M. (2010). Procesos cognitivos implicados en la lectura de niños

y niñas del tercer grado de educación primaria residentes en Lima y Piura. Revista de Investigación en Psicología. Volumen 13. $\mathrm{n}^{\circ}$ 1. Lima. Edic. Instituto de Investigaciones Psicológicas UNMSM

Flores, J. y Ostrosky, F. (2012). Desarrollo neuropsicológico de lóbulos frontales y funciones ejecutivas. México. Manual Moderno.

González, R. 2006. Problemas psicolingüisticos en el Perú. Obras Completas. Volumen I. Lima: Ediciones. N. Reátegui. 
Luria (1979), El cerebro en acción. Barcelona. Edit. Fontanella. teoría de Luria a niños a través de la Batería LURIA-DNI. Madrid. Edic. Visor.

Martínez, M. y Henao, G. (2006) Desempeño en la función ejecutiva de niños expuestos a ambientes de enseñanza bilingües. Electronic Journal of Research in Educational Psychology, vol. 4, núm. 3. España. Universidad de Almería.

Ortiz (2004). Introducción a la psicobiología del hombre. Lima. Fondo editorial UNMSM.

Papalia, D. y Wendkos, S. (1998) Psicología del desarrollo. Bogotá. Mc Graw-Hill.

Piaget, J. (1981) Psicología del niño. Madrid. Edic. Morata.

Quintanar, L. y Solovieva, Y. (2004) Evaluación Neuropsicológica Infantil. Lima. Perú Edic. Libro Amigo.

Rubia, K., Overmeyer, S., Taylor, E., Brammer, M., Williams, S. y Simmons, A. (2001).

Functional frontalisation with age: mapping neurodevelopment trajectories with fMRI. Neuroscience and Biobehavorial Review. 24 (1). 13-19

Sánchez, H. y Reyes, C. (2002) Metodología y diseños en la investigación científica. Lima. Edic. Universidad Ricardo Palma.

Velarde (2001) Relación entre la conciencia fonológica y el nivel de decodificación y comprensión lectora en niños de 8 años del 3er. Grado de primaria de dos niveles socio- económicos del cercado del Callao. Tesis de Maestría en educación. UNIFE. Lima. Perú.

Velarde, E. y Canales, R. 2013. La lectura en el Perú. Una salida a la crisis. Lima. Edic. Garabato.

Vigotsky, L. (1964) "Pensamiento y Lenguaje “. Buenos Aires. Editorial Lautaro.

Wechsler, D. (2006) WISC-IV Escala para la inteligencia de Wechsler para niños IV. Manual técnico de interpretación. Madrid. Edic. TEA. 